\title{
A Re-analysis of Caries Rates in a Preventive Trial using Poisson Regression Models
}

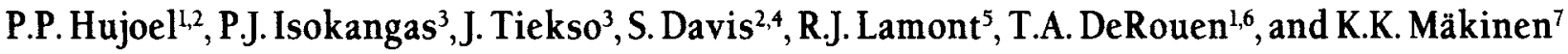 \\ 'Department of Dental Public Health Sciences, School of Dentistry, SM-35, University of Washington, Seattle, Washington 98195; ${ }^{2}$ Department \\ of Epidemiology, School of Public Health and Community Medicine; ${ }^{3}$ Department of Community Dentistry, Institute of Dentistry, University of \\ Turku, Finland; ${ }^{4}$ Fred Hutchinson Cancer Research Center, Division of Public Health Sciences, Program in Epidemiology, and University of \\ Washington, Seattle; Departments of ${ }^{5}$ Oral Biology and ${ }^{6}$ Biostatistics, University of Washington, Seattle; and ${ }^{7}$ Department of Biological and \\ Material Sciences, University of Michigan, Ann Arbor, Michigan 48109
}

\begin{abstract}
The analysis of caries incidence in clinical trials has several challenging features: (1) The distribution of the number of caries onsets per patient is skewed, with the majority of patients having few or no cavities; (2) the number of surfaces at risk varies (i) over time and (ii) between patients, due to eruption and exfoliation patterns, dental diseases, and treatments; (3) surfaces within a patient differ in their caries susceptibility, and (4) caries onsets within a patient arecorrelated due to shared host factors. Recent statistical developments in the area of correlated data analyses permit incorporation of some of these characteristics into the analyses. With Poisson regression models, the expected number of cariesonsets can be related to the number of surfaces at risk, the time they have been at risk, and surface- and subject-specific explanatory variables. The parameter estimated in these models is an epidemiological measure of disease occurrence: the disease incidence rate (caries rate) or the rate of change from healthy (sound) to diseased (carious). Differences and ratios of these rates provide standard epidemiological measures of excess risk. To illustrate, Poisson regression models were used for exploratory analyses of the Ylivieska xylitol study.
\end{abstract}

Key words. Dental Caries, Models, Statistical, Xylitol.

Received October 22, 1992; Accepted November 16, 1993

This investigation was supported in part by USPHS Research Grant 1 K11 DE0031 4 from the National Institute of Dental Research.

\section{Introduction}

The change $(\Delta)$ in the number of Decayed, Filled, and Missing teeth or surfaces (DMF) has commonly been used to measure caries incidence. If $\mathrm{DMF}_{\mathrm{li}}$ and $\mathrm{DMF}_{2 \mathrm{i}}$ are the caries scores in person $i$ at time points $\mathrm{l}$ and 2 , and $\mathrm{N}$ is the patient sample size, then the $\triangle \mathrm{DMF}$ score is:

$$
\Delta \mathrm{DMF}=\frac{\underset{\mathrm{i}=1}{\mathrm{~N}\left(\mathrm{DMF}_{2 \mathrm{i}}-\mathrm{DMF}_{1 \mathrm{i}}\right)}}{\mathrm{N}}
$$

This statistic, which estimates the mean population DMF score change, has certain limitations when used as a measure of disease incidence. Ideally, the calculation of disease occurrence should take into account the number of disease onsets, the size of the population at risk, and the time that the population was at risk. This information is not always present in the $\triangle \mathrm{DMF}$ score. The numerator of the $\triangle \mathrm{DMF}$ score reflects the number of caries onsets minus the number of remineralizations. If remineralizations dooccur, the $\triangle \mathrm{DMF}$ score underestimates the number of new disease onsets. The $\triangle D M F$ denominator counts one "person" unit for each person. As a result, both the number of surfacesand the timeat risk for each surface are not taken into account. Variations in the number of teeth at risk across patients and across time due to the teeth's natural eruption and exfoliation patterns, disease occurrences, or extractions are difficult to incorporate into the $\triangle \mathrm{DMF}$ scores.

The $\triangle D M F$ scores are usually analyzed with analysis of variance (ANOVA) methods (or derivatives thereof, such as Student's $t$ tests). With these methods, the total variability in caries scores, as measured by the sums of the squares of deviations from the mean, is divided into components associated with specific sources of variation, such as treatments (Marriott, 1990). 
Table 1. Calculation of time-at-risk and caries rates for four subjects

\begin{tabular}{|c|c|c|c|c|c|c|c|c|c|c|}
\hline \multirow[t]{2}{*}{ Subject } & \multirow{2}{*}{$\begin{array}{c}\text { Type of Surface } \\
j\end{array}$} & \multirow{2}{*}{$\begin{array}{l}\text { At-risk } \\
\text { surfaces }\end{array}$} & \multirow[t]{2}{*}{ Baseline } & \multirow[t]{2}{*}{ Exam 1} & \multirow[t]{2}{*}{ Exam 2} & \multicolumn{2}{|c|}{ \# Caries Onsets } & \multicolumn{2}{|c|}{ Time at Risk } & \multirow{2}{*}{$\begin{array}{c}\text { Caries Rates } \\
\text { (\# caries onset/ } \\
\text { surface-years) }\end{array}$} \\
\hline & & & & & & $\begin{array}{l}\text { lst } \\
\text { exam }\end{array}$ & $\begin{array}{l}\text { 2nd } \\
\text { exam }\end{array}$ & $\begin{array}{l}\text { lst } \\
\text { exam }\end{array}$ & $\begin{array}{l}\text { 2nd } \\
\text { exam }\end{array}$ & \\
\hline \multirow[t]{3}{*}{1} & Molar pit-and-fissures & 12 & OCT82 & NoV83 & OCT84 & 0 & 0 & $12 \times 1.08 \mathrm{yr}$ & $12 \times 0.92$ & $0 / 24=0$ \\
\hline & Other & $\underline{44}$ & & & & 0 & 0 & $44 \times 1.08 \mathrm{yr}$ & $44 \times 0.92$ & $0 / 88=0$ \\
\hline & All surfaces & $\overline{56}$ & & & & & & 60.48 & 51.52 & $0 / 112=0$ \\
\hline \multirow[t]{3}{*}{2} & Molar pit-and-fissures (E) & 21 & SEP82 & NOV83 & NOV84 & 0 & 2 & $21 \times 1.17 \mathrm{yr}$ & $19 \times 1+2 \times 0.5$ & $\begin{array}{l}52 / 44.6= \\
44.9 \times 10^{-3}\end{array}$ \\
\hline & Other & $\underline{104}$ & & & & 0 & 0 & $104 \times 1.17 \mathrm{yr}$ & $104 \times 1$ & $0 / 225.7=0$ \\
\hline & All surfaces & 125 & & & & & & 146.3 & 124 & $\begin{array}{l}2 / 270.3= \\
7.4 \times 10^{-3}\end{array}$ \\
\hline \multirow[t]{3}{*}{3} & Molar pit-and-fissures (E) & 19 & OCT82 & OCT83 & OCT84 & 4 & 1 & $15 \times 1+4 \times 0.5$ & $14 \times 1+1 \times 0.5$ & $\begin{array}{l}5 / 31.5= \\
158.7 \times 10^{-3}\end{array}$ \\
\hline & Other & $\underline{103}$ & & & & 10 & 2 & $93 \times 1+10 \times 0.5$ & $91 \times 1+2 \times 0.5$ & $512 / 190=$ \\
\hline & All surfaces & 122 & & & & & & 115 & 106.5 & $\begin{array}{l}63.2 \times 10^{-3} \\
17 / 221.5= \\
76.7 \times 10^{-3}\end{array}$ \\
\hline \multirow[t]{7}{*}{4} & Molar pit-and-fissures ( $\left.E^{a}\right)$ & 12 & OCT82 & OCT83 & OCT84 & 0 & 0 & $12 \times 1$ & $12 \times 1$ & $0 / 24=0$ \\
\hline & Molar pit-and-fissures $\left(\mathrm{El}^{\mathrm{b}}\right)$ & 3 & & & & 0 & 1 & $3 \times 0.5$ & $2 \times 1+1 \times 0.5$ & $\begin{array}{l}1 / 4= \\
250 \times 10^{-3}\end{array}$ \\
\hline & Molar pit-and-fissures $\left(E 2^{c}\right)$ & c) 9 & & & & - & 0 & - & $9 \times 0.5$ & $0 / 4.5=0$ \\
\hline & Other $(E)$ & 76 & & & & 0 & 0 & $76 \times 1$ & $76 \times 1$ & $0 / 152=0$ \\
\hline & Other (El) & 17 & & & & 0 & 0 & $17 \times 0.5$ & $17 \times 1$ & $0 / 25.5=0$ \\
\hline & Other (E2) & 6 & & & & - & 0 & & $6 \times 0.5$ & $0 / 3=0$ \\
\hline & All surfaces & $\overline{123}$ & & & & & & 98 & 115 & $\begin{array}{l}1 / 213= \\
4.7 \times 10^{-3}\end{array}$ \\
\hline
\end{tabular}

E: erupted prior to baseline.

bEl: erupted between baseline and exam 1.

'E2: erupted between exam 1 and exam 2 .

Some assumptions underlying this analytic approach fit caries data poorly. With ANOVA techniques, it is assumed that the DMF scores are normally distributed. Yet, it is well-known that disease counts, such as the number of caries onsets per patient, are usually highly skewed, with the majority of patients having no new lesions. Although such misf its have almost noeffects on the Type I error rates of the statistical test (Glass et al.,1972), they do affect one's ability to describe the caries data. Also, with ANOVA techniques the sample size is commonly assumed to be of fixed size over the study period. Thus, changes in the number of surfaces or teeth-at-risk changes due to eruptions or exfoliations are difficult to incorporate into the modeling of the data.

Recently, the National Institute of Dental Research recommended that analytical methods used in oral epidemiology should belinkedmoreclosely with those usedingeneralepidemiology(US Department of Health \& Human Services, 1990). Poisson regression models have been used extensively for the study of disease incidence. The assumptions underlying these modelsalso appear to be better suited for the analysis of caries incidence data than are
ANOVA models. By means of Poisson regression models (1) the skewed distribution of cariesonsetsin a population can be modeled as a Poisson random variable, rather than a normal random variable; (2) the changes in the number of surfaces at risk over time and between patients(eruptions, exfoliations, dentaldiseases, and treatments) can be taken into account, rather than a fixed size being assumed for the population at risk; and (3) both host factors-such as treatments assigned to patients-and local factors--such as the post-eruptive age of a surface-can be modeled.

The purposes of this study were to explore the Ylivieska (Finland)xylitol preventivetrial by meansof Poisson regression models and to discuss some of the benefits of the application of standard epidemiological methods to the analysis of caries incidence data.

\section{Materials and Methods}

\section{Data}

DMF-based results and design characteristics of the Ylivieska 
caries study have been described in two publications (Isokangas et al., 1988, 1989). Briefly, the caries-preventive effectiveness of xylitol gum was investigated with a prospective cohort design. A total of 324 children wasfollowed over an approximate two-year period (range, 1.9-2.3 years) and examined annually between 1982 and 1984. Children in the xylitol group $(n=172$; mean age $=$ $11.5 ; \mathrm{SD}=0.5 ; 84$ males) were instructed to chew xylitol gum 3 times daily. No supervised gum program was present in the control group $(n=152$ children; mean age $=115 ; \mathrm{SD}=0.5 ; 82$ males $)$.

\section{Statistical analysis}

The purpose was to study how the risk for developing a caries lesion on a sound surface differed between the xylitol and control groups. The data for this question were set up in the following manner: For the category of surfaces having characteristic $j$ within patient $i$, the number of incident caries lesions $\left(\mathrm{y}_{\mathrm{ij}}\right)$ and the surface-time at risk $\left(\mathrm{T}_{\mathrm{ij}}\right)$ were calculated. Since over $60 \%$ of all caries onsets were located on molars as pit-andfissure caries, the surfaces were stratified as (1) molar pit-andfissures ( 3 surfaces per molar) vs. (2) other surfaces. The caries onsets and the number of surfaces at risk for four subjects are displayed in Table 1. For instance, subject 1 had zero caries onsets during the two-year study and 56 surfaces at risk (caries-free at baseline). A surface diagnosed as carious or restored at baseline was never included in the analysis (even if it was classified as sound at subsequent visits).

At each visit subsequent to the baseline, three events can be distinguished for the "at risk" surfaces:

Event A: Surface remained caries-free. For instance, subject 1 had 12 molar pit-and-fissure surfaces which remained caries-free both between baseline and exam 1 (a time period of 1.08 years), and between exams 1 and 2 (a time period of 0.98 years). The time at risk for molar pitand-fissure caries in subject 1 was 13 surface-years for period 1 ( $12 \times 1.08)$ and 11 surface-years for period 2 . For subject 4, 3 molar pit-and-fissure surfaces erupted between baseline and visit 1 . Since the exact eruption time was unknown, we assumed that eruption occurred in the middle of the observation period. Thus, the timeat risk for these 3 surfaces during period 1 was $3 \times 0.5$ years or 1.5 surface-years.

Event B: Surface developed caries. For instance, in subject 3, 4 out of 19 molar pit-and-fissure surfaces diagnosed as sound at baseline developed caries between baseline and visit 1 . The exact time these 4 surfaces developed caries was unknown. By convention, we assumed that the caries onset occurred in the middle of the first observation period. Thus, for subject 3,4 surfaces were at risk for $1 / 2$ year, and 15 surfaces were at risk for 1 year, leading to a total of 17 years at risk between baseline and visit 1 .

In the present analyses, the definition of event $B$ was limited to the incidence of the "first" caries occurrence on a surface. Such an approach appears reasonable in young populations, where most surfaces were sound at the start of the study and the incidence of first caries occurrences constituted the majority of events of interest. When a surface developed caries, it was removed from the "at risk" population for the remainder of the study (even if it was diagnosed at a subsequent visit as sound again). The manner in which the "at risk" population and event Bare defined may be modified according to the study's purpose. For instance, in older populations, where recurrent caries lesions can be of importance, the definition of a caries onset can be modified to accommodate the study of recurrent caries lesions.

Event C: Surface lost due to extraction or exfoliation (a potential source of bias). In the present study, there were no surfaces diagnosed as sound at baseline which were coded as extracted during the two-year study. Surfaces of subjects dropping out of the study stop contributing time at the last dental exam.

In general, the time at risk for events $A, B$, and $C$ was calculated as follows: If the time between two examinations for patient $i$ was $t_{i}$, the time contributed for a surface was $t_{i}$ for event $A$, and $t_{i} / 2$ for events $B$ and $C$. When a surface erupted between the yearly examinations, it contributed $t_{i} / 2$ for event $A$, and $t_{i} / 4$ for events $B$ and $C$. The surface-time at risk for surfaces with characteristic $j$ within patient $i$ is the sum of all the surfacetimes $\left(t_{i}\right)$ of surfaces with characteristic $j$ or $T_{i j}$.

With this described methodology, only caries-free sound surfaces contributed to $T_{i j}$ Stated differently, only surfaces which had a non-zero chance of developing a first caries onset were included. Surfaces which had caries or were restored at baseline, or surfaces which remained unerupted during the study, were excluded from the analyses. (Exclusion of sealed surfaces was impossible for the presented data. The presence of sealants was notreported on the clinicalforms.) A commonquestion regarding this analytical approach is why only sound surfaces are selected for the analyses. For cohort studies on disease incidence, only those participants of a population who are at risk for developing the disease of interest are eligible. For instance, in a prevention trial for coronary heart disease, one of the eligibility criteria was theabsence of heart disease (Sherwinetal.,1981). Potential participants in this study were screened on two different occasions to exclude patients with clinical evidence of coronary heart disease or other cardiovascular diseases. Similarly, in the analysis of caries incidence trials, one of the ligibility criteria for a surface to be included in the analysis should be the absence of clinical evidence of caries. Surfaces diagnosed as carious at baseline or with evidence of past caries (a filling) should be excluded from the analyses if the purpose is to study the causal factors responsible for primary caries onsets on sound surfaces.

After the number of carious onsets and the time surfaces were at risk are determined, the caries rate can be estimated. The ratio of the number of incident caries lesions to the sum of all surface-years at risk provides an unbiased estimate of the caries rate. Caries rates are interpreted as a change per unit time (Kelsey et al., 1986). The caries rate represents the estimated rate of change from sound to carious. For instance, subject 3 in Table 1 had 17 caries onsets and 221.5 surface-years at risk during the two-year study, leading to an estimated caries rate of 76.7 caries onsets per 1000 surface-years ob- 
served (the highest observed caries rate for this cohort).

The definitions of events $A, B$, and $C$ are associated with measurement error. Examiners differ in their criteria as to what constitutes a caries lesion, whether restorations traverse the demarcation boundary of an adjacent tooth surface, and also on tooth position. Some surfaces diagnosed as sound are in fact carious, or vice versa. In the analyses of some clinical trials, caries reversals were considered a diagnostic error, and different statistical methods were developed to adjust for this measurement error (Espeland et al, 1988; Carlos and Senning, 1968; Reef and McHugh, 1977). While such models may lessen the impact of misclassification, the validity of such adjustments depends on the validity of the biological rationale that cariesisan irreversible process. Analternative common approach to misclassification in epidemiological studies is to analyze the data "as is" and toevaluate how they affect the findings of the study. If the misclassification biases of the clinicians are similar in the different study groups, which can be achieved by means of a doubleblind randomized controlled trial, the studied association (e.g., sugar and caries) is biased toward the null hypothesis (Kleinbaum et al, 1982).

The purpose of the statistical analyses was to identify those explanatory variables which have a substantial impact on the caries rates. In thisstudy, the explanatory variables savailable for each group of surfaces $j$ within patient $i\left(\mathrm{X}_{\mathrm{ijs}}\right)$ are:

Treatment: xylitol group, control group.

Gender: male, female.

Study period: Fall, 1982-Fall, 1983;

Fall 1983-Fall, 1984.

Patient's age: age in years.

Post-eruptive surface age: surface-age in years (Table 2).

Surface characteristics: Molar pit-and-fissure surfaces $v s$. "other" surfaces.

The statistical models relating the caries incidence rates to the explanatory variables are called Poisson regression models and are of the following form:

$$
\log \left(\mathrm{y}_{\mathrm{ij}}\right)=\log \left(\mathrm{T}_{\mathrm{ij}}\right)+\alpha+\Sigma\left(\beta_{\mathrm{s}} \mathrm{X}_{\mathrm{ij}}\right)
$$

where $\alpha$ is the intercept and $\beta_{\mathrm{s}}$ is the slope coefficient associated with the explanatory variable $\mathrm{X}_{\mathrm{ijs}}$ (McCullagh and Nelder, 1989). What do the estimated coefficients of the Poisson regression model (1) tell us about the investigated explanatory variables and their relationship to caries rates? Exponentiating $\alpha$ provides an estimate of the caries reference rate, $\boldsymbol{\kappa}_{\mathrm{o}}$. It expresses the expected number of lesions for a fixed number of surfaceyears of observation. Its dimensionality, like any other rate, is $1 /$ time. To specif $y$ what type of time is being measured, the word "time" is preceded by the unit of analysis. Since the unit of analysis in model (1) is a surface, the dimensionality of the rate is $1 /$ surface-time. Exponentiating $\beta_{s}\left(e^{\beta_{5}}\right)$ providesanestimate of the rate ratio; it explainson a multiplicative scale how the caries reference rate, $\boldsymbol{\kappa}_{0}$, varies as a function of the explanatory vari-

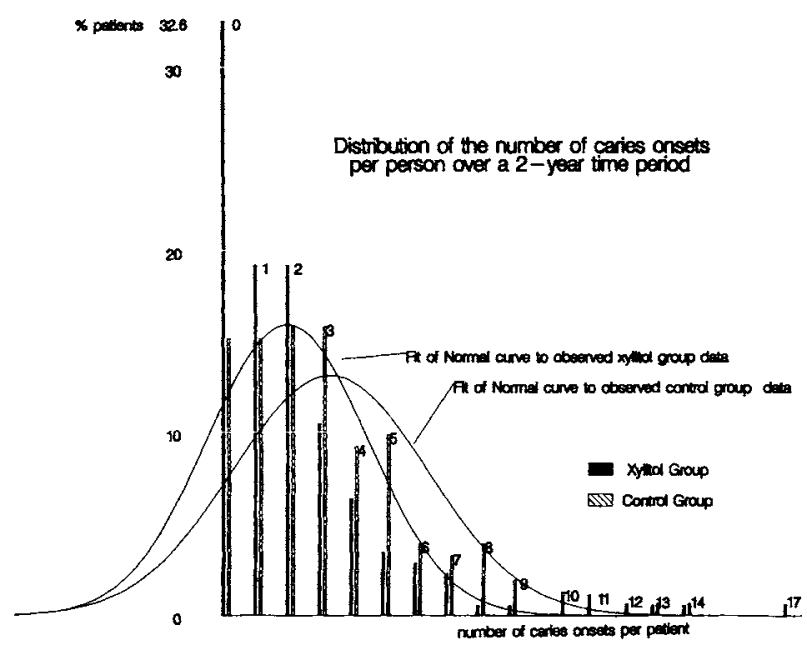

Figure 1. Histogram of the number of caries onsets per patient stratified by treatment group.

able. For instance, if $e^{\beta_{s}}$ equals 2 , it indicates that the caries rate doubles for every unit increase of $X_{s}$.

Estimating the parameters $\alpha$ and $\beta_{\mathrm{s}}$ of model (1) is complex due to the correlation of caries occurrences within patients. The standard methods of estimation are based on the assumption that events occur independently of each other. This assumption of independence is not valid for the study of caries. Most of the cariesactivity usually occursin a small subset of the population. In other words, there is a larger variability in caries rates among patients than would be expected based on the assumption of Poisson independence. The excess variability due to correlation of events is commonly called "extra-Poisson variability". Several approaches to deal with this extra-Poisson variability have recently been suggested (Breslow, 1984; Liang and Zeger, 1986; Campbell et al., 1991). In this study, the parameters were estimated by generalized estimating equations (Liang and Zeger, 1986). Generalized estimating equations are an extension of the multiple-regression model to a class of maximum likelihood procedures (Marriott, 1990). The statistical significance of each parameter was assessed by the Wald statistic.

\section{Results}

The distribution of the number of caries onsets per patient differed substantially between the xylitol and the control groups(Fig.1).In the xylitol group, over $70 \%$ of the children had 0,1 , or 2 lesions. In the control group, the distribution was less skewed, with more children exhibiting a larger number of new lesions. A normal curve was fitted to the data for visual display of the fit that would be obtained by assuming that the number of caries onsets was distributed as a normal random variable. For both the xylitol and control groups, the normal curve fitted poorly. Thefit of the normal distribution, for instance, indicated that a significant percentage of the patients had a negative 
number of new caries occurrences on a scale which, by definition, can consist of only positive integers. Subjects in the xylitol and control groups had, on average, 218 and 217 surface-yearsat risk, respectively (Fig. 2). There was no significant difference in the average surface time-at-risk between the two groups (twosample t test; $\mathrm{p}=0.88$ ). Note, however, the large variability in the number of surface-years at risk between patients (Fig. 2). The number of surface-years at risk differed significantly between males and females (two-sample t test: $p<0.01$ ). Females, due to earlier eruption patterns, had, on average, 15 more surface-years at risk than males. In the next paragraphs, the influences of different explanatory variables on caries incidence rates are explored by means of Poisson regression models.

\section{Treatment as sole explanatory variable}

The caries rates differed signif icantly between the xylitol and the control groups $(\mathrm{p}<0.01)$. In the control group, 15.5 caries lesions occurred per 1000 surfaces observed per year. In the xylitol group, 9.3 caries lesions occurred per 1000 surfaces observed per year. Thus, the caries rate was 1.7 times higher in the control than in the xylitol group.

\section{Treatment, gender, and patient's age as explanatory variables}

In addition to treatment, both patient's age (Wald statistic, $\mathrm{p}<$ 0.01 ) and gender (Wald statistic; $p<0.01$ ) substantially affected caries risk. Caries risk decreased with increasing age and was higher for females than males. Xylitol's effectiveness may have depended on patient's gender (gender-treatment interaction). When compared with the control group, females in the xylitol group were 2.2 times less likely to have a caries onset, whereas males in the xylitol group were only 1.4 times less likely to have a caries onset (Wald statistic of gendertreatment interaction term; $\mathrm{p}=0.09$ ).

\section{Treatment, post-eruptive surf ace age, and} study period as explanatory variables

When post-eruptive age was included in the model, the analy-
Distribution of the surface-years at risk per person over a 2-year time period

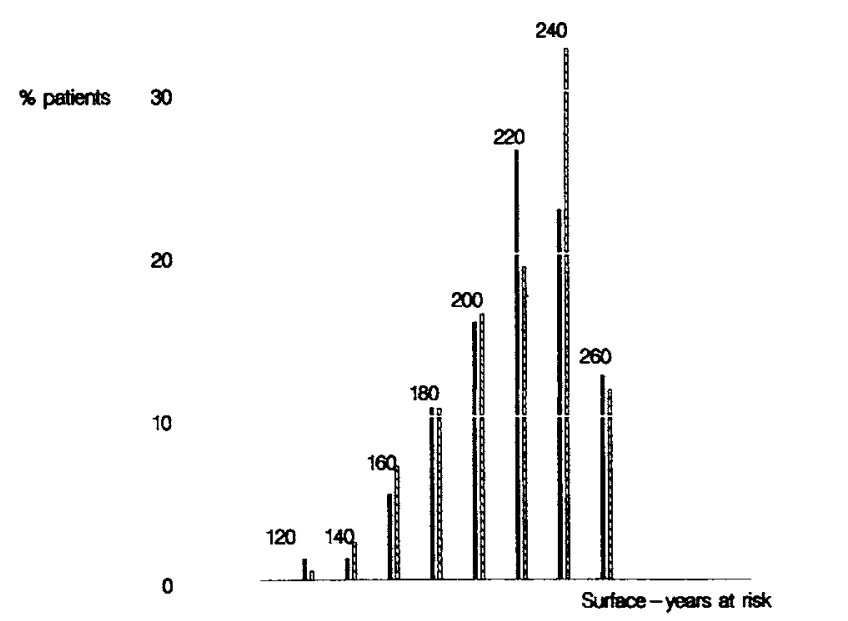

Figure 2. Histogram of the number of surface-yearsat risk per patient stratified by treatment group.

ses were limited to molar pit-and-fissure caries (Table 2). Posteruptive pit-and-fissure age had an effect on xylitol effectiveness during the second study year (Wald statistic; $\mathrm{p}=0.04$ ). During the second study year, xylitol gum was 2 times more effective in preventing caries on molar pits and fissures less than 1 year old than on molar pits and fissures more than 2 yearsold. Study period also had a significant impact on xylitol effectiveness. For pit-and-fissure surfaces less than 1 year old, the xylitol gum was 3.1 times more effective in preventing caries during the second year of the study than it was during the first year ( $95 \%$ c.i.: $1.0-9.5$ ) (Wald statistic; $p=0.048$ ). No consistent gender effects on xylitol effectiveness were present when the analyses were adjusted for post-eruptive age.

\section{Discussion}

The results of these exploratory analyses confirmed the previously reported findings:Incorporation of xylitol gum intoacaries-preven-

Table 2. Estimation of Post-eruptive Age of second molars

\begin{tabular}{|c|c|c|c|c|}
\hline Study Period & Eruption Time & No. Teeth & Tooth Age & $\begin{array}{c}\text { Post-eruptive Age } \\
\text { Estimate }\end{array}$ \\
\hline \multirow[t]{3}{*}{$1982-1983$} & unerupted & 302 & & \\
\hline & $' 82-' 83$ & 353 & Surface less than 1 year old & 0.5 \\
\hline & prior to' 82 & 641 & Surface older than 1 year & $1.5-?^{2}$ \\
\hline \multirow[t]{4}{*}{ 1983-1984 } & unerupted & 88 & & \\
\hline & $83-84$ & 214 & Surface less than 1 year old & 0.5 \\
\hline & '82-'83 & 353 & Surface between 1 and 2 years old & 1.5 \\
\hline & prior to ' 82 & 641 & Surface older than 2 years & $2.5-?$ \\
\hline
\end{tabular}

a Since these teeth erupted before the study started, the post-eruptive surface age could not be determined with the same precision as the posteruptive surface age of teeth which erupted during the study. For this reason, post-eruptive surface age was treated as an ordinal variable, rather than a continuous variable. 
tive program resulted in a significant caries reduction. In the xylitol group, 9.3 caries lesions were diagnosed per 1000 surfaces observed per year. In the control group, 155 caries lesions were diagnosed per 1000 surfacesobserved peryear. The rateat which new carieslesions appeared in the control group was 1.7 times higher than that in the xylitol group. During exploratory analyses, two potential xylitol effect modifiers were identified: a period effect and a post-eruptive age effect (It should be emphasized that the results of these exploratory analyses serve only to generate hypothesesfor further studies, and they should not be interpreted as "definitive" findings.)

\section{Period effect}

For surfaces less than 1 year old, xylitol was 3.1 times more effective in preventing caries during the second year of the study than it wasduring thef irst year. Possibly, there wasa lag between the start of the xylitol program and the onset of the preventive effects. Many preventive programs are associated with a lag period. For instance, the benefits of a reduction of serum cholesterol level for the prevention of coronary heart disease may not be notable for many years (Cornfield and Mitchell, 1969). The benefits of smoking cessation for the prevention of mortality in older men may not be visible for a decade (Cornfield and Mitchell, 1969). Similarly, it may be that there is a lag, of smaller magnitude, between the start of the xylitol program and the achievement of its full preventive effect on caries rates.

\section{Post-eruptive age effect}

During the second year of the study, the xylitol effectiveness varied substantially as a function of post-eruptive age:Xylitol was most effective for pits and fissures less than 1 year old, and its effectiveness decreased with increasing post-eruptive age. Both the caries risk (Carlos and Gittlesohn, 1965) and the xylitol effectiveness were strongly related to post-eruptive age. On a multiplicative scale, when the risk for caries was high, xylitol effectiveness was high, and when the risk for caries was low, xylitol effectiveness was low. These findings support the proposed hypothesis that the maturation of teeth underf avorable physico-chemical conditions has a long-term beneficial effect (Isokangas et al., 1989). Use of xylitol chewing gum during the high-risk, early, post-eruptive period allows surfaces to mature under favorable conditions. Cessation of gum use after this high-risk period still results in permanent benefits, since the surfaces are now at a lower risk for caries.

Several of the key characteristics of the analytical approach presented in this study have been suggested in the past. The concept of estimating the time at risk for each individual surface and calculating a "true" caries-risk measure using the life-table method was discussed in detail by Carlos and Gittelsohn (Carlos and Gittelsohn, 1965). Further, the fitting of Poisson models to caries data was already evaluated in 1954 (Grainger and Reid, 1954). Thanks to recent advances in the theory of generalized linear models, two elements were added to this earlier work: (1) modeling of the variability in caries rates between persons, and (2) linear models-Poisson regression modeling of the variability in caries rates as a function of covariates. These additions increase the ease by which these methods can be applied. With the life-table methodology, the caries rates had to be estimated for each tooth separately, and the effects of covariates had to be evaluated by means of stratification. With over-dispersed Poisson regression models, the variability of caries rates on different surfaces can be modeled as a function of covariates within one model. With the increasing availability of sof tware, this approach could be as routine to implement as the standard DMF-based ANOVA methods.

The DMF-based ANOVA-based analyses and Poisson regression models differ in several respects:(1) the definition of the sample; (2) the concept of "at risk" time; (3) the evaluation of age and period effects; and (4) the resulting measures of excess risk. In the following paragraphs, these differences are emphasized. (Both ANOVA and Poisson regression models are variations of linear models; consequently, the differences between models may sometimes have more to do with the way these two data analytical approaches have been used, rather than with their intrinsic properties.)

\section{The definition of the sample}

When caries incidence data are modeled with Poisson regression models, only surfaces with well-defined characteristics are included in the analysis (e.g., sound surfaces at risk for developing caries). Subsequently, causal factors influencing the onset of caries are investigated. In DMF-based ANOVA analyses, both sound and carious surfaces are included in the analyses, and as a result, the effect of the intervention on existing caries lesions (treatment effects) and the effect of the intervention on sound surfaces (preventive effects) are usually not separated in the analyses.

\section{The "time at risk" concept}

It is statistically inefficient to describe caries by counting the number of caries onsets and assuming that the resulting score is distributed as a normal random variable (assumption of ANOVA models). Counts are usually better approximated by assuming that the underlying probability model is a Poisson random variable. In addition, to estimate a rate, it is important to know how many surfaces were at risk and how long they had been at risk. Without estimation of the time at risk, one cannot calculate disease rates.

\section{The evaluation of age, period, and cohort effects}

Disease rates may vary as a function of age (either patient's or post-eruptive surface age), calendar year (period), or birth year (cohort). Since most diseases are strongly influenced by age, and less so by study period, incidence rates are calculated by methods that make allowances for age-specific changes and period changes (Case and Lea, 1955). The calculation of caries rates involved estimating the surface-years at risk experienced at different post-eruptive ages, and also making allowance for the date to which the post-eruptive age experience referred. It is more difficult to take such effects into account 
with DMF-based ANOVA analyses. ANOVA analyses are usually applied to study groups with a fixed sample size (a fixed population), and the standard epidemiological methods used for measurement of time at risk for different age categories and different periods are difficult to incorporate.

\section{The resulting measures of excess risk}

Another difference between Poisson regression models and DMF-based ANOVA models is the resulting measures of treatment efficacy. Poisson regression models provide commonly used epidemiological disease measures such as disease incidence rates and rate ratios. ANOVA models provide mean changes of caries scores. Means as a summary statistic are notorious for misinterpretation and have been referred to as "inappropriate measures of disease association" in general epidemiology (Breslow and Day, 1980).

In summary, Poisson regression methods may offer advantages over DMF-based ANOVA analyses. Poisson regression methodsallow for the estimation of a fundamental measure of disease occurrence: the disease incidence rate or the rate at which new events occur in a population in a given time period. For each individual surf ace, the time at risk isestimated, and as a result changes in the number of surfaces at risk or the time they have been at risk are incorporated into the estimation of the disease rates. This property appears usef ul for caries studies which are typically performed in populations where the number of teeth at risk can vary considerably both across patients and across time. The new conclusions that can be drawn from this re-analysis were that for molar pit-and-fissure caries, (1) there may be a lag effect for the beneficial effects of xylitol gum, and (2) the xylitol effectiveness may be dependent on the post-eruptive age. It is concluded that the application of standardepidemiological methods to theanalyses of caries trials can potentially increase the amount of information that can be extracted from the data,

\section{References}

Breslow NE (1984). Extra-Poisson variability in log-linear modeling. Appl Statist 33:38-44.

Breslow NE, Day NE(1980). Measures of disease. In:Statistical methods in cancer research. Vol. 1. The analysis of case-control studies. Lyon: IARC Scientific Publications, 66.

Campbell MJ, Machin D, D'Arcangues C (1991). Coping with extraPoisson variability in the analysis of factors influencing vaginal ring expulsions. Stat Med 10:241-254.

Carlos JP, Gittlesohn AM (1965). Longitudinal study of the natural history of caries. Il. A life-table study of caries incidence in the permanent teeth. Arch Oral Biol 10:739-751.

Carlos JP, Senning RS (1968). Error and bias in dental clinical trials.] Dent Res 67:142-148.

Case RAM, Lea AJ (1955). Mustard gas poisoning, chronic bronchitis, and lung cancer. An investigation into the possibility that poisoning by mustard gas in the 1914-18 war might be a factor in the production of neoplasia. BrJ Prev Soc Med 9:62-72.

Cornfield J, Mitchell S (1969). Selected risk factors in coronary heart disease. Possible intervention effects. Arch Environ Hlth 19:382-394

Espeland MA, Murphy WC, Leverett DH (1988). Assessing diagnostic reliability and estimating incidence ratesassociated with a strictly progressive disease: Dental caries. Stat in Med 7:403-416.

Glass RL, Alman JE, Fleisch S, D'Agostino RB (1972). The appropriateness of analysis of variance to the statistical analyses of dental clinical trials. Arch Oral Biol 17:633-643.

Grainger RM, Reid DBW (1954). Distribution of dental caries in children.J Dent Res 33:613-623.

Isokangas P, Alanen P, Tiekso J, Măkinen KK(1988).Xylitol chewing gum in caries prevention: a field study in children. JADA 117:315-320.

Isokangas P, Alanen P, Tiekso J, Mäkinen KK (1989). Long-term effect of xylitol chewing gum on dental caries. Community Dent Oral Epidemiol 17:200-203.

Kelsey JL, Thompson WD, Evans AS (1986). Biological and statistical concepts. In:Methods in observational epidemiology. Monographs in epide miology and biostatistics. Vol. 10. Oxford: University Press, 23-45.

Kleinbaum DG, Kupper LL, Morgenstern H (1982). Epidemiologic research. Principles and quantitative methods. New York: Van Nostrand Reinhold Company.

Liang KY, Zeger SL (1986). Longitudinal data analysis using generalized linear models. Biometrika 73:13-22

Marriott FHC, editor (1990). A dictionary of statistical terms. Essex: Longman Scientific and Technical.

McCullagh P, Nelder JA (1989). Generalized linear models. 2nd ed London: Chapman and Hall.

Reed GF, McHugh R (1979). The estimation of dental caries incidence in the presence of diagnostic error. Biometrics 35:473-478.

Sherwin R, Kaelber CT, Kezdi P, Kjelsberg MO, Thomas HE(1981). The multiple risk intervention trial (MRFIT). II. The development of the protocol. Prev Med 10:402-425.

US Department of Health and Human Services (1990). New findings/ new directions. In: Broadening the scope. National Institute of Dental Research, 134. 